\title{
ANATOMY OF NONCOSTAL PORTIONS OF LAMINA IN THE CYCLANTHACEAE (MONOCOTYLEDONEAE). $V$. TABLES OF DATA
}

\section{GEORGE J. WiLDER*}

This is the last paper of a series which pertains to lamina anatomy in the Cyclanthaceae (Wilder, in press a, b, c, d). Each paper is based on the same fifty-three species and ten genera of the family. Collection localities have been listed (Tomlinson and Wilder, 1984). The present paper includes tables which document findings in all previous papers of the series. Tables are organized into four groups representing paper nos. $I-I V$ of the series, respectively. Each group of tables is introduced by a synopsis of the paper which it represents.

\section{INTRODUCTION TO TABLES $1-6$}

(Wilder, in press $a$ )

The outer walls of ordinary epidermal cells exhibit inner noncutinized and outer cutinized regions, and are covered by a cuticle sensu stricto (Table 1). Noncostal portions of cyclanthaceous laminae are always hypostomatic, i.e., having the majority of stomata situated in the abaxial epidermis (Tables 2, 3 ). In noncostal parts of this epidermis, stomata are oriented within stomatal bands, and such bands are separated by interstomatal bands of four main kinds: $(1)$ in interridge areas, bands located over superficially situated fiber strands of the mesophyll, (2) also in interridge areas, bands situated over the largest longitudinal veins, (3) bands occurring on abaxial ridges, and (4) bands of epidermal expansion tissue. Given a small strip of epidermis, it is sometimes possible to ascertain the following: whether it is adaxial or abaxial; if abaxial, whether portions thereof are from interridge areas, ridges, or expansion tissue, and

\footnotetext{
*Harvard Forest, Harvard University

Petersham, Massachusetts 01366

Present address: Cleveland State University, Dept. of Biology,

1983 East 24th Street, Cleveland, Ohio 44115
} 
which portions from interridge areas occur over fiber strands and the largest veins; which are the abaxial and adaxial sides of the strip; which are its distal and basal ends, and its left and right sides relative to the lamina it came from. A cyclanthaceous stoma is normally encircled by four subsidiary cells, and the stoma and associated subsidiary cells are collectively called a stomatal complex (Tables 4-6). Subsidiary cells may differ from ordinary epidermal cells in numerous ways such as their shape and position, nature of inclusions, staining of outer cell walls, size of nuclei, and thickness and ornamentation of the cuticle. In four species the stomata exhibit polar perforations, i.e., large pores located within the distal and basal ends of the common wall between twc ssociated guard cells. Cyclanthaceous stomata exhibit substantial dorsiventral symmetry.

\section{TABLE 1.}

GENERAL RANGE IN COMBINED THICKNESS OF CUTICLE AND CUTINIZED REGION (rounded off to nearest quarter micrometer)

\begin{tabular}{|c|c|c|}
\hline Species & Adaxial epidermis & Abaxial epidermis \\
\hline $\begin{array}{l}\text { A. aff. A. antioquiae } \\
\text { (coll. A) }\end{array}$ & $0.75-1.5$ & $0.5-2$ \\
\hline $\begin{array}{l}\text { A. aff. A. antioquiae } \\
\text { (coll. B) }\end{array}$ & $1-1.5$ & $0.5-1$ \\
\hline A. cabrerae & $2-3.25$ & $0.75-2.5$ \\
\hline A. cayapensis & $1.5-2.5$ & $0.5-2$ \\
\hline $\begin{array}{l}\text { A. sp. nov. aff. } A . \\
\quad \text { cupulifera (coll. A) }\end{array}$ & $1-1.5$ & $0.5-1$ \\
\hline $\begin{array}{l}\text { A. sp. nov. aff. } A \text {. } \\
\text { cupulifera (coll. B) }\end{array}$ & $3-4.75$ & $1-3.75$ \\
\hline A. gamotepala & $3.25-7.25$ & $1.5-6.25$ \\
\hline A. hookeri & $0.75-1.5$ & $<0.5-0.75$ \\
\hline A. longitepala & $1.5-2.5$ & $0.75-1.5$ \\
\hline $\begin{array}{l}\text { A. sp. nov. aff. } A . \\
\text { longitepala }\end{array}$ & $2.5-5.25$ & $1.5-4.25$ \\
\hline A. moritziana & $2-3.75$ & $0.75-3$ \\
\hline A. aff. A. moritz; & $3-4.25$ & $1-2.5$ \\
\hline
\end{tabular}


TABLE 1 (continued)

GENERAL RANGE IN COMBINED THICKNESS OF CUTICLE AND CUTINIZED

REGION (rounded off to nearest quarter micrometer)

\begin{tabular}{|c|c|c|}
\hline Species & Adaxial epidermis & Abaxic :piderm \\
\hline $\begin{array}{l}\text { A. sp. nov. aff. } A . \\
\text { multistaminata }\end{array}$ & $1-2.5$ & $0.5-1.5$ \\
\hline A. peruviana & $1.5-4.75$ & $0.5-2.5$ \\
\hline A. pycnantha & $2.5-4.5$ & $1-3$ \\
\hline A. quinindensis & $1-2.5$ & $0.5-1$ \\
\hline $\begin{array}{l}\text { A. sp. nov. aff. } A \text { : } \\
\text { rhodea }\end{array}$ & $1.5-2.5$ & $0.5-2$ \\
\hline A. rigida & $1.5-3$ & $0.5-1.25$ \\
\hline A. tetragona & $1.5-3.75$ & $0.75-1.5$ \\
\hline A. urophylla & $3-8.75$ & $1.5-4.75$ \\
\hline $\begin{array}{l}\text { A. aff. A. vaupesiana } \\
\quad \text { (coll. A) }\end{array}$ & $1.5-3.25$ & $0.5-2$ \\
\hline $\begin{array}{l}\text { A. aff. A. vaupesiana } \\
\text { (coll. B) }\end{array}$ & $1.5-2.5$ & $0.5-1.5$ \\
\hline $\begin{array}{l}\text { A. (Asplundia) } \\
\text { sp. nov. }\end{array}$ & $1-3$ & $1-3.25$ \\
\hline Ca. palmata & $3.5-5.5$ & $1-4$ \\
\hline Cy. bipartitus & $1-2$ & $0.5-1.5$ \\
\hline D. crinitum & $1.5-3.5$ & $1-2.5$ \\
\hline D. dolichostemon & $0.5-1.5$ & $<0.5-\quad .5$ \\
\hline D. globosum & $1.5-2.5$ & $1-3$ \\
\hline D. grandifolium & $0.5-1$ & $0.5-2$ \\
\hline D. harlingii & $<0.5-0.5$ & $<0.5-0.5$ \\
\hline D. macrophyllum & $1.5-5.75$ & $0.75-3.25$ \\
\hline D. mirabile & $0.75-1.5$ & $0.5-1.5$ \\
\hline $\begin{array}{l}\text { D. sp. nov. aff. } \\
\text { D. nanum }\end{array}$ & $1.5-3$ & $1-2.5$ \\
\hline D. rheithrophilum & $1.5-3.75$ & $1-3.75$ \\
\hline D. schultesii & $0.75-2.5$ & $0.5-3.25$ \\
\hline D. wallisii & 0.5 & $0.25-0.5$ \\
\hline D. sp. nov. (coll. A) & & $0.5-0.75$ \\
\hline D. sp. nov. (coll. B) & $0.5-1$ & 0.5 \\
\hline
\end{tabular}


TABLE 1 (continued)

GENERAL RANGE IN COMBINED THICKNESS OF CUTICLE AND CUTINIZED REGION (rounded off to nearest quarter micrometer)

\begin{tabular}{|c|c|c|}
\hline Species & Adaxial epidermis & Abaxial epidermis \\
\hline E. funifer & $2.0-3.5$ & $1-3.5$ \\
\hline L. bierhorstii & $1-3$ & $0.5-1$ \\
\hline L. integrifolia & $3.75-6.75$ & $2.5-5.25$ \\
\hline L. Iancifolia & $3-5$ & $2.5-5$ \\
\hline Sch. chorianthum & $1-1.5$ & $<0.5-0.5$ \\
\hline Sph. acutitepala & $3-4.75$ & $1-7.25$ \\
\hline Sph. crocea & $3.25-7.75$ & $1.5-4.75$ \\
\hline Sph. killipii & $5-7.5$ & $0.5-3$ \\
\hline Sph. snidernii & $6.25-13$ & $1-3.75$ \\
\hline Sph. woodsonii & $5.25-7.25$ & $0.5-1$ \\
\hline $\begin{array}{l}\text { Sph. sp. nov. aff. } \\
\text { Sph. woodsonii }\end{array}$ & $3-5.25$ & $1.5-3.25$ \\
\hline Sph. sp. nov. & $3.25-6.25$ & $0.5-3$ \\
\hline St. anomala & $7.75-12$ & $3.75-12$ \\
\hline St. stylaris & $8.5-12.5$ & $4-10.5$ \\
\hline T. bissectus & $1-6.5$ & $1-5$ \\
\hline
\end{tabular}


TABLE 2

CONCENTRATION OF STOMATA AND LAMINA THICKNESS IN INTERRIDGE AREAS.*

\begin{tabular}{|c|c|c|c|c|c|c|}
\hline \multirow[b]{2}{*}{$\begin{array}{l}\text { A. aff. A. antioquiae } \\
\text { (coll. A) }\end{array}$} & \multicolumn{3}{|c|}{$\begin{array}{c}\text { No. of stomata } / \mathrm{mm}^{2} \text { in the } \\
\text { abaxial epidermis, adaxial } \\
\text { epidermis, and both } \\
\text { epidermides }\end{array}$} & \multirow{2}{*}{$\begin{array}{l}\begin{array}{l}\text { Ratio of nos. of } \\
\text { stomata } / \mathrm{mm}^{2}- \\
\text { abaxial:adaxial }\end{array} \\
53\end{array}$} & \multirow{2}{*}{$\begin{array}{c}\begin{array}{c}\text { Lamina thickness - } \\
\text { general range and } \\
\text { midpoint of range } \\
(\mathrm{mm})\end{array} \\
0.18-0.29 ; 0.24\end{array}$} & \multirow{2}{*}{$\begin{array}{c}\begin{array}{c}\text { Lamina thickness } \\
\text { in nos. of cell } \\
\text { layers }\end{array} \\
11-13\end{array}$} \\
\hline & 213 & 4.0 & 217 & & & \\
\hline $\begin{array}{l}\text { A. aff. A. antioquiae } \\
\text { (coll. B) }\end{array}$ & 267 & 2.8 & 270 & 95 & $0.23-0.34 ; 0.29$ & $12-15$ \\
\hline A. cabrerae & 108 & 13 & 121 & 8.4 & $0.41-0.52 ; 0.46$ & $17-20$ \\
\hline A. cayapensis & 155 & 3.1 & 158 & 50 & $0.31-0.45 ; 0.38$ & $19-22$ \\
\hline $\begin{array}{l}\text { A. sp. nov. aff. } A \text {. } \\
\text { cupulifera } \\
\text { (coll. A) }\end{array}$ & 131 & 0.31 & 132 & 421 & $0.16-0.20 ; 0.18$ & $10-15$ \\
\hline $\begin{array}{l}\text { A. sp. nov. aff. } A \text {. } \\
\text { cupulifera } \\
\text { (coll. B) }\end{array}$ & 169 & 0.31 & 169 & 542 & $0.20-0.29 ; 0.24$ & $13-15$ \\
\hline A. gamotepala & 260 & 9.0 & 269 & 29 & $0.35-0.44 ; 0.40$ & $16-19$ \\
\hline A. hookeri & 117 & 3.1 & 120 & 38 & $0.22-0.29 ; 0.26^{* *}$ & $10-12^{* *}$ \\
\hline A. longitepala & 138 & 2.5 & 140 & 55 & $0.25-0.36 ; 0.30$ & $11-14$ \\
\hline $\begin{array}{l}\text { A. sp. nov. aff. } A \text {. } \\
\text { longitepala }\end{array}$ & 176 & 5.6 & 181 & 31 & $0.31-0.39 ; 0.35$ & $15-18$ \\
\hline A. moritziana & 118 & 4.7 & 122 & 25 & $0.23-0.29 ; 0.26$ & $12-16$ \\
\hline
\end{tabular}


TABLE 2 (continued)

CONCENTRATION OF STOMATA AND LAMINA THICKNESS IN INTERRIDGE AREAS.*

\begin{tabular}{|c|c|c|c|c|c|c|}
\hline \multirow[b]{2}{*}{ A. aff. A. moritziana } & \multicolumn{3}{|c|}{$\begin{array}{l}\text { No. of stomata } / \mathrm{mm}^{2} \text { in the } \\
\text { abaxial epidermis, adaxial } \\
\text { epidermis, and both } \\
\text { epidermides }\end{array}$} & \multirow{2}{*}{$\begin{array}{l}\begin{array}{l}\text { Ratio of nos. of } \\
\text { stomata } / \mathrm{mm}^{2}-\end{array} \\
\text { abaxial:adaxial }\end{array}$} & \multirow{2}{*}{ 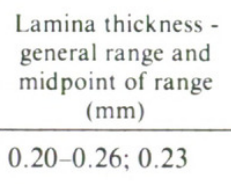 } & \multirow{2}{*}{$\begin{array}{c}\begin{array}{c}\text { Lamina thickness - } \\
\text { in nos. of cell } \\
\text { layers }\end{array} \\
11-14\end{array}$} \\
\hline & 269 & 1.2 & 270 & & & \\
\hline $\begin{array}{l}\text { A. sp. nov. aff. } A \\
\text { multistaminata }\end{array}$ & 144 & 12 & 156 & 12 & $0.21-0.27 ; 0.24$ & $11-13$ \\
\hline A. peruviana & 167 & 8.3 & 175 & 20 & $0.21-0.27 ; 0.24$ & $11-12$ \\
\hline A. pycnantha & 166 & 4.0 & 170 & 41 & $0.30-0.37 ; 0.33$ & $16-20$ \\
\hline A. quinindensis & & & & & $0.19-0.26 ; 0.23$ & $11-13$ \\
\hline $\begin{array}{l}\text { A. sp. nov. aff. } \\
\text { A. rhodea }\end{array}$ & 156 & 0.93 & 157 & 167 & $0.20-0.23 ; 0.22$ & $11-12$ \\
\hline A. rigida & 132 & 5.6 & 138 & 24 & $0.28-0.37 ; 0.32$ & $14-15$ \\
\hline A. tetragona & 140 & 5.6 & 145 & 25 & $0.19-0.24 ; 0.22$ & $12-15$ \\
\hline A. urophylla & 207 & 8.1 & 215 & 26 & $0.24-0.32 ; 0.28$ & $15-18^{* *}$ \\
\hline $\begin{array}{l}\text { A. aff. A. vaupesiana } \\
\text { (coll. A) }\end{array}$ & 188 & 11 & 199 & 17 & $0.23-0.29 ; 0.26^{* *}$ & $12-15^{* *}$ \\
\hline $\begin{array}{l}\text { A. aff. A. vaupesiana } \\
\text { (coll. B) }\end{array}$ & 107 & 8.4 & 116 & 13 & $0.30-0.34 ; 0.32$ & $13-15$ \\
\hline $\begin{array}{l}\text { A. (Asplundia) } \\
\text { sp. nov. }\end{array}$ & 104 & 3.4 & 107 & 30 & $0.32-0.41 ; 0.36$ & $12-14$ \\
\hline
\end{tabular}




Ca. palmata
C.. bipartitus
D. crinitum
D. dolichostemon
D. globosum
D. grandifolium
D. harlingii
D. macrophyllum
D. mirabile
D. sp. nov. aff. D.
nanum
D. rheithrophilum
D. schultesii
D. wallisii
D. sp. nov.
(coll. A)
D. sp. nov.
(coll. B)
E. funifer
L. bierhorstii
L. integrifolia
L. lancifolia
Sch. chorianthum

$\begin{array}{ccc}269 & 1.2 & 270 \\ 311 & 41 & 352 \\ 234 & 0.93 & 235 \\ 142 & 29 & 170 \\ 261 & 0 & 261 \\ 107 & 30 & 138 \\ 108 & 46 & 154 \\ 255 & 0.31 & 255 \\ 165 & 62 & 228 \\ & & \\ 270 & 26 & 296 \\ 189 & 19 & 208 \\ 151 & 28 & 179 \\ 273 & 22 & 295 \\ 71 & 8.1 & 79 \\ & & \\ 194 & 9.0 & 203 \\ & & \\ 160 & 14 & 174 \\ 59 & 2.5 & 61 \\ 91 & 26 & 117 \\ 44 & 34 & 78 \\ 144 & 0 & 144\end{array}$

$\begin{array}{clc}215 & 0.18-0.26 ; 0.22 & 12-16 \\ 7.6 & 0.26-0.41 ; 0.33 & 14-16 \\ 250 & 0.34-0.43 ; 0.39 & 20-27 \\ 4.9 & 0.23-0.31 ; 0.27 & 14-16 \\ \text { ca. } \infty & 0.32-0.37 ; 0.35 & 12-14 * * \\ 3.6 & 0.19-0.26 ; 0.22 & 9-12 \\ 2.3 & & \\ 818 & 0.34-0.45 ; 0.40 & 19-21 \\ 2.7 & 0.23-0.32 ; 0.28 & 11-14 \\ 10 & 0.27-0.33 ; 0.30 & 12-14 * * \\ 10 & 0.41-0.48 ; 0.44 & 14-18 \\ 5.4 & 0.19-0.24 ; 0.22 & 10-14 \\ 12 & 0.25-0.28 ; 0.27 & 12-15 * * \\ 8.7 & 0.28-0.33 ; 0.30 & 9-11 \\ & & \\ 21 & 0.28-0.36 ; 0.32 & 14-16 \\ 11 & 0.17-0.24 ; 0.20 & 10-13 \\ 24 & 0.26-0.32 ; 0.29 * * & 10-12 * * \\ 3.5 & 0.37-0.46 ; 0.42 & 12-15 \\ 1.3 & 0.47-0.61 ; 0.54 & 12-17 \\ \text { ca. } \infty & 0.12-0.16 ; 0.14 & 8-9\end{array}$


TABLE 2 (continued)

CONCENTRATION OF STOMATA AND LAMINA THICKNESS IN INTERRIDGE AREAS.*

\begin{tabular}{|c|c|c|c|c|c|c|}
\hline \multirow[b]{2}{*}{ Sph acutitepala } & \multicolumn{3}{|c|}{$\begin{array}{c}\text { No. of stomata } / \mathrm{mm}^{2} \text { in the } \\
\text { abaxial epidermis, adaxial } \\
\text { epidermis, and both } \\
\text { epidermides }\end{array}$} & \multirow{2}{*}{$\begin{array}{l}\text { Ratio of nos. of } \\
\text { stomata/mm² } \\
\text { abaxial:adaxial } \\
\text { ca. } \infty\end{array}$} & \multirow{2}{*}{ 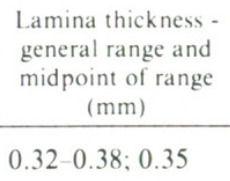 } & \multirow{2}{*}{$\begin{array}{c}\begin{array}{c}\text { Lamina thickness } \\
\text { in nos. of cell } \\
\text { layers }\end{array} \\
15-17\end{array}$} \\
\hline & 236 & 0 & 236 & & & \\
\hline Sph. crocea & 164 & 0.62 & 164 & 262 & $0.29-0.48 ; 0.39$ & $16-18$ \\
\hline Sph. killipii & 309 & 5.6 & 315 & 55 & $0.33-0.38 ; 0.36$ & $16-18$ \\
\hline Sph. snidernii & 273 & 10 & 283 & 27 & $0.28-0.36 ; 0.32$ & $15-17$ \\
\hline Sph. woodsonii & 188 & 0 & 188 & ca. $\infty$ & $0.36-0.42 ; 0.39$ & $16-17$ \\
\hline $\begin{array}{l}\text { Sph. sp. nov. aff. } \\
\text { Sph. woodsonii }\end{array}$ & 123 & 3.7 & 127 & 33 & $0.40-0.45 ; 0.43$ & 22 \\
\hline Sph. sp. nov. & 486 & 1.9 & 488 & 260 & $0.23-0.32 ; 0.27$ & $12-14$ \\
\hline St. anomala & 105 & 0 & 105 & ca. $\infty$ & $0.30-0.41 ; 0.36^{* *}$ & $13-17 * *$ \\
\hline St. stylaris & 176 & 1.9 & 178 & 94 & $0.47-0.61 ; 0.54$ & $18-22$ \\
\hline T. bissectus & 227 & 80 & 307 & 2.8 & $0.33-0.43 ; 0.38$ & $17-20$ \\
\hline
\end{tabular}

*Stomatal frequencies were determined by studying mostly stained, but also unstained epidermal peels with the phase microscope at $100 \mathrm{X}$. Stomata were counted on $3.21 \mathrm{~mm}^{2}$ of a peel from each of the abaxial and adaxial epidermides, with the following exceptions (the two figures between each pair of parentheses indicate areas studied [ $\left.\mathrm{mm}^{2}\right]$ of the abaxial and adaxial epidermides, respectively): A. peruviana (1.81, 1.81), A. urophy./la (3.01, $3.21)$. D. harlingii $(2.01,3.21)$, and D. mirahile $(2.01,1.61)$. As often as possible on a peel, fields were studied which were laterally rather than longitudinally adjacent to one another, to maximize randomness of sampling. i.e., to avoid including the same stomatal or interstomatal band(s) in all samples. For certain species the concentration indicated for both epidermides is slightly different from the total of the separate indicated concentrations, because the total was computed and rounded off. prior to rounding off of the separate values. Lamina thickness in mm was determined only for cross sections. Thickness in numbers of cell layers was ascertained, using portions of longitudinal sections containing only epidermal cells and ordinary parenchyma cells.

**These values were obtained from study of unembedded material which was bleached in an aqueous solution of sodium hypochlorite, and mounted in glycerine. Remaining values were determined during study of stained, plastic-embedded material. 
TABLE 3

STOMATAL RATIOS IN THE INTERRIDGE AREA*

\begin{tabular}{lccc}
\hline & $\begin{array}{c}\text { Percentage of species with } \\
\text { low ratios }(0-29)\end{array}$ & $\begin{array}{c}\text { Percentage of species with } \\
\text { moderate ratios (30-99) }\end{array}$ & $\begin{array}{c}\text { Percentage of species with } \\
\text { high ratios (100 and higher) }\end{array}$ \\
\hline $\begin{array}{l}\text { Asplundia }(22,94) \\
\text { Carludovica }(1,3)\end{array}$ & 45.5 & 36.4 & 18.2 \\
Cyclanthus $(1,1)$ & - & - & 100 \\
Dicranopygium $(13,48)$ & 100 & - & - \\
Evodianthus $(1,1)$ & 100 & - & 23.1 \\
Ludovia $(3,3)$ & 100 & - & - \\
Schultesiophytum $(1,1)$ & 14.3 & 50 & 100 \\
Sphaeradenia $(7,42)$ & - & - & 57.1 \\
Stelestylis $(2,4)$ & 100 & 50 \\
Thoracocarpus $(1,1)$ & - & - \\
* In parentheses after the name of each genus are indicated the number of species considered, and the total number of species known, not including \\
undescribed species collected by the writer.
\end{tabular}




\section{TABLE 4}

PERCENTAGES OF STOMATAL COMPLEXES IN THE ABAXIAL EPIDERMIS IN WHICH NEITHER, ONE, OR BOTH NONPOLAR SUBSIDIARY CELl(s) EXTEND(s) TO THE OUTER EDGE(S) OF THE ASSOCiATED CELL File(s).*

\begin{tabular}{|c|c|c|c|c|}
\hline & $\begin{array}{l}\text { Neither extends } \\
\text { to outer edge }\end{array}$ & $\begin{array}{l}\text { One extends to } \\
\text { outer edge }\end{array}$ & $\begin{array}{c}\text { Both extend to } \\
\text { outer edge }\end{array}$ & $\begin{array}{c}\text { One or both extend } \\
\text { to outer edge }(\mathrm{s}) \\
\text { (column } 2+\text { column } 3 \text { ) }\end{array}$ \\
\hline $\begin{array}{l}\text { A. aff. A. antioquiae } \\
\text { (coll. A) }\end{array}$ & 76.7 & 20.0 & 3.3 & 23.3 \\
\hline $\begin{array}{l}\text { A. aff. A. antioquiae } \\
\text { (coll. B) }\end{array}$ & 85.3 & 13.3 & 1.3 & 14.7 \\
\hline A. cabrerae & 24.0 & 47.3 & 28.7 & 76.0 \\
\hline A. cayapensis & 94.7 & 5.3 & 0.0 & 5.3 \\
\hline $\begin{array}{l}\text { A. sp. nov. aff. } A \text {. } \\
\text { cupulifera } \\
\text { (coll. A) }\end{array}$ & 85.3 & 14.7 & 0.0 & 14.7 \\
\hline $\begin{array}{l}\text { A. sp. nov. aff. } A \text {. } \\
\text { (upulifera } \\
\text { (coll. B) }\end{array}$ & 95.3 & 4.7 & 0.0 & 4.7 \\
\hline A. gamotepala & 67.3 & 28.0 & 4.7 & 32.7 \\
\hline A. hookeri & 96.7 & 3.3 & 0.0 & 3.3 \\
\hline A. longitepala & 78.7 & 20.7 & 0.7 & 21.3 \\
\hline $\begin{array}{l}\text { A. sp. nov. aff. } A \text {. } \\
\text { longitepala }\end{array}$ & 75.3 & 22.7 & 2.0 & 24.7 \\
\hline A. moritziana & 86.0 & 13.3 & 0.7 & 14.0 \\
\hline
\end{tabular}




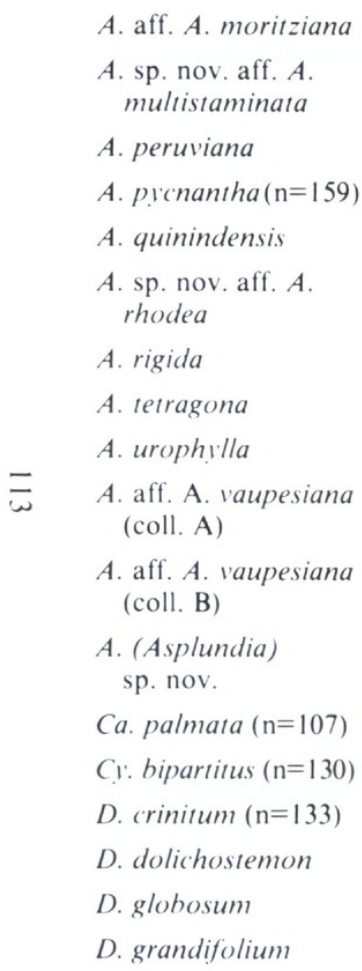


TABLE 4 (continued)

PERCENTAGES OF STOMATAL COMPLEXES IN THE ABAXIAL EPIDERMIS IN WHICH NEITHER, ONE, OR BOTH NONPOLAR SUBSIDIARY CELL(S) EXTEND(S) TO THE OUTER EDGE(S) OF THE ASSOCIATED CELL FILE(S).*

\begin{tabular}{|c|c|c|c|c|}
\hline & $\begin{array}{l}\text { Neither extends } \\
\text { to outer edge }\end{array}$ & $\begin{array}{l}\text { One extends to } \\
\text { outer edge }\end{array}$ & $\begin{array}{l}\text { Both extend to } \\
\text { outer edge }\end{array}$ & $\begin{array}{l}\text { One or both extend } \\
\text { to outer edge(s) } \\
\text { (column } 2+\text { column } 3 \text { ) }\end{array}$ \\
\hline D. harlingii & 100 & 0 & 0 & 0 \\
\hline D. macrophyllum & 95.3 & 4.7 & 0.0 & 4.7 \\
\hline D. mirabile & 96.7 & 3.3 & 0.0 & 3.3 \\
\hline $\begin{array}{l}\text { D. sp. nov. aff. } D \text {. } \\
\text { nanum }\end{array}$ & 91.3 & 8.7 & 0.0 & 8.7 \\
\hline D. rheithrophilum & 90.0 & 9.3 & 0.7 & 10.0 \\
\hline D. schultesii & 93.3 & 6.7 & 0.0 & 6.7 \\
\hline D. wallisii & 99.3 & 0.7 & 0.0 & 0.7 \\
\hline $\begin{array}{l}\text { D. sp. nov. } \\
\text { (coll. A) }\end{array}$ & 100.0 & 0.0 & 0.0 & 0.0 \\
\hline $\begin{array}{l}\text { D. sp. nov. } \\
\text { (coll. B) }\end{array}$ & 92.0 & 8.0 & 0.0 & 8.0 \\
\hline E. funifer $(\mathrm{n}=122)$ & 45.1 & 39.3 & 15.6 & 54.9 \\
\hline L. bierhorstii & 92.0 & 7.3 & 0.7 & 8.0 \\
\hline L. integrifolia & 18.0 & 49.3 & 32.7 & 82.0 \\
\hline L. Iancifolia $(\mathrm{n}=134)$ & 28.4 & 43.3 & 28.4 & 71.7 \\
\hline Sch. chorianthum & 96.0 & 4.0 & 0.0 & 4.0 \\
\hline
\end{tabular}




\begin{tabular}{|c|c|c|c|c|}
\hline Sph. acutitepala & 2.0 & 26.0 & 72.0 & 98.0 \\
\hline Sph. crocea & 1.3 & 31.3 & 67.3 & 98.7 \\
\hline Sph. killipii $(\mathrm{n}=151)$ & 15.2 & 48.3 & 36.4 & 84.7 \\
\hline $\begin{array}{l}\text { Sph. snidernii } \\
\quad(\mathrm{n}=151)\end{array}$ & 39.1 & 46.4 & 14.6 & 60.9 \\
\hline Sph. noodsonii & 47.3 & 40.0 & 12.7 & 52.7 \\
\hline $\begin{array}{l}\text { Sph. sp. nov. aff. } \\
\text { Sph. noodsonii }\end{array}$ & 24.7 & 47.3 & 28.0 & 75.3 \\
\hline Sph. sp. nov. & 53.3 & 40.0 & 6.7 & 46.7 \\
\hline St. anomala & 8.0 & 37.3 & 54.7 & 92.0 \\
\hline St. stylaris $(\mathrm{n}=131)$ & 11.5 & 47.3 & 41.2 & 88.5 \\
\hline T. bissectus $(\mathrm{n}=129)$ & 25.6 & 45.0 & 29.5 & 74.5 \\
\hline
\end{tabular}




\section{TABLE 5}

PERCENTAGES OF SPECIES IN WHICH LOW, MODERATE, OR HIGH PERCENTAGES OF COMPLEXES EXHIBIT ONE OR TWO NONPOLAR SUBSIDIARY CELL(S) WHICH EXTEND TO THE OUTER EDGE(S) OF THE ASSOCIATED CELL FILE(S)*

\begin{tabular}{lccc}
\hline & $\begin{array}{c}\text { Percentage of species with } \\
\text { low percentages of } \\
\text { such complexes }(1-11 \%)\end{array}$ & $\begin{array}{c}\text { Percentage of species with } \\
\text { moderate percentages of } \\
\text { such complexes (12-44\%) }\end{array}$ & $\begin{array}{c}\text { Percentage of species with } \\
\text { high percentages of } \\
\text { such complexes }(45-99 \%)\end{array}$ \\
\hline $\begin{array}{l}\text { Asplundia }(23,94) \\
\text { Carludovica }(1,3)\end{array}$ & $21.7 \%$ & $69.6 \%$ & $8.7 \%$ \\
Cyclanthus $(1,1)$ & - & - & $100 \%$ \\
Dicranopygium $(13,48)$ & $100 \%$ & - & $100 \%$ \\
Evodianthus $(1,1)$ & - & - & - \\
Ludovia $(3,3)$ & $33.3 \%$ & - & $100 \%$ \\
Schultesiophytum $(1,1)$ & $100 \%$ & - & $-6.6 \%$ \\
Sphaeradenia $(7,42)$ & - & - & $100 \%$ \\
Stelestylis $(2,4)$ & - & - & $100 \%$ \\
Thoracocarpus $(1,1)$ & - & - & $100 \%$
\end{tabular}

*In parentheses after the name of each genus are indicated the number of species considered, and the total number of species known, not including undescribed species collected by the writer. 


\section{TABLE 6}

CONTACTS BETWEEN STOMATAL COMPLEXES

\begin{tabular}{|c|c|c|c|c|}
\hline & $\begin{array}{l}\text { Percentage of stomatal } \\
\text { complexes in contact } \\
\text { with one or more other } \\
\text { stomatal complexes }\end{array}$ & $\begin{array}{c}\text { Percentage of stomatal } \\
\text { complexes which } \\
\text { share subsidiary } \\
\text { cells }\end{array}$ & $\begin{array}{l}\text { Percentage of contacts } \\
\text { which entail sharing } \\
\text { of subsidiary cells } \\
\text { (column } 2 / \text { column } 1 \text { ) }\end{array}$ & $\begin{array}{l}\text { Types of sharing of } \\
\text { subsidiary cells by } \\
\text { two adjacent stomatal } \\
\text { complexes* }\end{array}$ \\
\hline Asplundia pycnantha & $\begin{aligned} & 50.3 \%(\mathrm{n}=150 \\
&\text { complexes })\end{aligned}$ & $\begin{array}{l}2 \%(\mathrm{n}=200 \\
\quad \text { complexes })\end{array}$ & $4.0 \%$ & $\begin{array}{l}\text { Type 1: } 100 \%(n=2 \\
\text { pairs of complexes })\end{array}$ \\
\hline Carludovica palmata & $64.2 \%(n=151)$ & - & - & - \\
\hline Cyclanthus bipartitus & $97.0 \%(n=131)$ & $11 \%(n=200)$ & $11.3 \%$ & $\begin{array}{c}\text { Type 1: } 54.5 \% \\
\text { Type 2: } 27.3 \% \\
\text { Type 3: } 18.2 \% \\
(n=11)\end{array}$ \\
\hline crinitum & $49.1 \%(n=159)$ & $2 \%(n=200)$ & $4.1 \%$ & $\begin{array}{l}\text { Type 1: } 100 \% \\
(n=2)\end{array}$ \\
\hline Evodianthus funifer & $47.7 \%(n=155)$ & $0 \%(n=200)$ & $0 \%$ & $0 \%$ \\
\hline Ludovia lancifolia & $4 \% \quad(n=200)$ & $0 \%(n=200)$ & $0 \%$ & $0 \%$ \\
\hline $\begin{array}{l}\text { Schultesiophytum } \\
\text { chorianthum }\end{array}$ & $66.9 \%(n=154)$ & $2 \%(n=200)$ & $3.0 \%$ & $\begin{array}{l}\text { Type 1: } 50 \% \\
\text { Type 2: } 50 \% \\
\quad(n=2)\end{array}$ \\
\hline $\begin{array}{c}\text { killipii } \\
\text { Stelestylis }\end{array}$ & $65.4 \%(n=153)$ & $7 \%(n=200)$ & $10.7 \%$ & $\begin{array}{l}\text { Type 1: } 100 \% \\
(n=7)\end{array}$ \\
\hline $\begin{array}{l}\text { stylaris } \\
\text { Thoracocarpus }\end{array}$ & $70.9 \%(n=182)$ & $7 \%(n=200)$ & $9.9 \%$ & $\begin{array}{l}\text { Type } 1: 85.7 \%(n=7) \\
\text { Type } 3: 14.3 \%\end{array}$ \\
\hline bissectus & $78.3 \%(n=143)$ & $6 \%(n=200)$ & $7.7 \%$ & $\begin{array}{c}\text { Type 1: } 33.3 \% \\
\text { Type 3: } 66.6 \% \\
(n=6)\end{array}$ \\
\hline
\end{tabular}

\footnotetext{
*Types of sharing are defined by Wilder (in press $a$ ).
} 
Within interridge areas and between boundary layers, portions of cyclanthaceous laminae exhibit either two (adaxial and abaxial) or three main regions of mesophyll (adaxial, middle, and abaxial; Table 7). The adaxial region is only sometimes a palisade region, whereas, the middle and abaxial regions are spongy mesophyll. Regions of mesophyll are distinguished mostly according to features of ordinary parenchyma cells. These cells may exhibit various ergastic materials, including starch, tannin, and different kinds of crystals. In certain species some cells also contain star figures, i.e., small or large stellate inclusions tentatively interpreted as tannin. In most species ordinary parenchyma cells are essentially monomorphic, but in two species of Dicranopygium these cells exhibit pronounced dimorphism (Table 8). Fibers occur in the mesophyll of all species studied, but differ quantitatively between various species (Tables 9-11). Parenchyma-like dead cells were observed in several and all species of the Asplundia group and Sphaeradenia group, respectively, but only such cells of the Sphaeradenia group exhibited conspicuously birefringent cell walls. Those dead cells with birefringent walls, therefore, constitute an extremely important systematicanatomical character within the Cyclanthaceae.

\footnotetext{
* Table no. 8 is included in Wilder (in press $b$ ), where it is listed as Table 1.
} 


\section{TABLE 7}

SPECIES WITH THREE MAIN REGIONS OF MESOPHYLL

(ALL UNLISTED SPECIES HAVE TWO REGIONS).

A. cabrerae

A. cayapensis

A. gamotepala

A. moritziana

A. aff. A. moritziana

A. pycnantha

$A$. sp. nov. aff. $A$. rhodea

A. tetragona

A. aff. A. vaupesiana (coll. $A$ )

Cy. bipartitus

E. funifer

L. lancifolia

Sph. snidernii

Sph. sp. nov. aff. Sph. woodsonii

St. stylaris

T. bissectus

TABLE 9

DATA PERTAINING TO FIBER STRANDS OF THE MESOPHYLL

\begin{tabular}{ccc}
\hline Species & $\begin{array}{c}\text { No. of fiber strands } / \mathrm{mm} \\
\text { width of interridge area } \\
\text { (in parentheses are indicated } \\
\text { no. of strands counted and } \\
\text { width of portion(s) of } \\
\text { lamina considered in } \mathrm{mm}, \\
\text { respectively) }\end{array}$ & $\begin{array}{c}\text { Percentage of fiber strands } \\
\text { on adaxial half of interridge } \\
\text { area (in parentheses are } \\
\text { indicated no. of strands } \\
\text { counted on adaxial and } \\
\text { both sides of lamina, } \\
\text { respectively }\end{array}$ \\
\hline
\end{tabular}

A. aff. A. antioquiae (coll. $A$ )

$29(189,6.64)$

$55.0(104,189)$

$A$. aff. A. antioquiae (coll. B)

$28 \quad(203,7.24)$

$64.5(131,203)$

A. cabrerae

$83(224,2.72)$

$60.3(135,224)$

A. cayapensis

$49(119,2.45)$

$59.7(71,119)$ 
TABLE 9 (continued)

DATA PERTAINING TO FIBER STRANDS OF THE MESOPHYLL

\begin{tabular}{|c|c|c|c|}
\hline Species & \multicolumn{2}{|c|}{$\begin{array}{l}\text { No. of fiber strands } / \mathrm{mm} \\
\text { width of interridge area } \\
\text { (in parentheses are indicated } \\
\text { no. of strands counted and } \\
\text { width of portion(s) of } \\
\text { lamina considered in mm, } \\
\text { respectively) }\end{array}$} & $\begin{array}{c}\text { Percentage of fiber strands } \\
\text { on adaxial half of interridge } \\
\text { area (in parentheses are } \\
\text { indicated no. of strands } \\
\text { counted on adaxial and } \\
\text { both sides of lamina, } \\
\text { respectively }\end{array}$ \\
\hline $\begin{array}{l}\text { A. sp. nov. aff. } A . \\
\quad \text { cupulifera (coll. } A)\end{array}$ & 39 & $(311,8.01)$ & $54.0(168,311)$ \\
\hline $\begin{array}{l}\text { A. sp. nov. aff. } A . \\
\text { cupulifera (coll. } B)\end{array}$ & 78 & $(510,6.54)$ & $55.5(283,510)$ \\
\hline A. gamotepala & 93 & $(210,2.26)$ & $56.7(119,210)$ \\
\hline A. hookeri & 17 & $(94,5.58)$ & $48.9(46,94)$ \\
\hline A. longitepala & 18 & $(78,4.44)$ & $69.2(54,78)$ \\
\hline $\begin{array}{l}\text { A. sp. nov. aff. } A . \\
\text { longitepala }\end{array}$ & 48 & $(224,4.70)$ & $55.8(125,224)$ \\
\hline A. moritziana & 26 & $(196,7.45)$ & $52.0(102,196)$ \\
\hline A. aff. A. moritziana & 20 & $(119,5.93)$ & $58.0(69,119)$ \\
\hline $\begin{array}{l}\text { A. sp. nov. aff. } A \text {. } \\
\text { multistaminata }\end{array}$ & 24 & $(91,3.83)$ & $58.2(53,91)$ \\
\hline A. peruviana & 46 & $(205,4.41)$ & $61.5(126,205)$ \\
\hline A. pycnantha & 65 & $(327,5.06)$ & $55.0(180,327)$ \\
\hline A. quinindensis & 49 & $(232,4.73)$ & $56.5(131,232)$ \\
\hline $\begin{array}{l}A . \text { sp. nov. aff. } A \text {. } \\
\text { rhodea }\end{array}$ & 32 & $(99,3.06)$ & $57.6(57,99)$ \\
\hline A. rigida & 28 & $(172,6.13)$ & $57.6(99,172)$ \\
\hline A. tetragona & 38 & $(158,4.19)$ & $51.9(82,158)$ \\
\hline A. urophylla & 19 & $(121,6.55)$ & $61.2(74,121)$ \\
\hline $\begin{array}{l}\text { A. aff. } A . \text { vaupesiana } \\
\quad(\text { coll. } A)\end{array}$ & 92 & $(366,4.00)$ & $63.7(233,366)$ \\
\hline $\begin{array}{l}\text { A. aff. A. vaupesiana } \\
\quad(\text { coll. } B)\end{array}$ & 24 & $(127,5.23)$ & $70.1(89,127)$ \\
\hline $\begin{array}{l}\text { A. (Asplundia) } \\
\text { sp. nov. }\end{array}$ & 28 & $(127,4.61)$ & $59.2(109,184)$ \\
\hline Ca. palmata & 66 & $(480,7.26)$ & $61.7(296,480)$ \\
\hline Cy. bipartitus & & $(46,8.00)$ & $97.8(45,46)$ \\
\hline D. crinitum & 40 & $(176,4.41)$ & $55.1(97,176)$ \\
\hline
\end{tabular}


TABLE 9 (continued)

DATA PERTAINING TO FIBER STRANDS OF THE MESOPHYLL

\begin{tabular}{|c|c|c|}
\hline Species & $\begin{array}{l}\text { No. of fiber strands } / \mathrm{mm} \\
\text { width of interridge area } \\
\text { (in parentheses are indicated } \\
\text { no. of strands counted and } \\
\text { width of portion(s) of } \\
\text { lamina considered in } \mathrm{mm} \text {, } \\
\text { respectively) }\end{array}$ & $\begin{array}{c}\text { Percentage of fiber strands } \\
\text { on adaxial half of interridge } \\
\text { area (in parentheses are } \\
\text { indicated no. of strands } \\
\text { counted on adaxial and } \\
\text { both sides of lamina, } \\
\text { respectively }\end{array}$ \\
\hline D. dolichostemon & $13(48,3.63)$ & $58.3(28,48)$ \\
\hline D. globosum & $13 \quad(93,7.27)$ & $46.2(43,93)$ \\
\hline D. grandifolium & $12 \quad(55,4.66)$ & $58.2(32,55)$ \\
\hline D. harlingii & $4.3(23,5.31)$ & $100 \quad(36,36)$ \\
\hline D. macrophyllum & $27 \quad(111,4.12)$ & $36.0(40,111)$ \\
\hline D. mirabile & $5.7(31,5.46)$ & $71.0(22,31)$ \\
\hline $\begin{array}{l}D . \text { sp. nov. aff. } D \text {. } \\
\text { nanum }\end{array}$ & $9.5(89,9.05)$ & $96.6(86,89)$ \\
\hline D. rheithrophilum & $24 \quad(153,6.37)$ & $56.2(86,153)$ \\
\hline D. schultesii & $20 \quad(99,4.91)$ & $59.6(59,99)$ \\
\hline D. wallisii & $19 \quad(139,7.49)$ & $64.7(90,139)$ \\
\hline$D$. sp. nov. $($ coll. $A)$ & $8.0(79,9.83)$ & $50.6(40,79)$ \\
\hline D. sp. nov. (coll. B) & $17 \quad(132,7.59)$ & $44.7(59,132)$ \\
\hline E. funifer & $46 \quad(154,3.39)$ & $56.1(83,148)$ \\
\hline L. bierhorstii & $13(67,5.25)$ & $82.1(55,67)$ \\
\hline L. integrifolia & $29 \quad(101,3.47)$ & $53.5(54,101)$ \\
\hline L. Iancifolia & $75 \quad(482,6.41)$ & $57.1(275,482)$ \\
\hline Sch. chorianthum & $24 \quad(110,4.62)$ & $64.9(72,111)$ \\
\hline Sph. acutitepala & $56 \quad(333,5.91)$ & $50.5(168,333)$ \\
\hline Sph. crocea & $27 \quad(276,10.2)$ & $56.5(156,276)$ \\
\hline Sph. killipii & $73 \quad(456,6.25)$ & $50.2(229,456)$ \\
\hline Sph. snidernii & $30 \quad(171,5.65)$ & $53.8(92,171)$ \\
\hline Sph. woodsonii & $108 \quad(209,1.93)$ & $58.9(123,209)$ \\
\hline $\begin{array}{l}\text { Sph. sp. nov. aff. } \\
\text { Sph. woodsonii }\end{array}$ & $49 \quad(308,6.26)$ & $66.2(204,308)$ \\
\hline Sph. sp. nov. & $30 \quad(188,6.27)$ & $41.0(77,188)$ \\
\hline St. anomala & $41 \quad(185,4.48)$ & $60.0(111,185)$ \\
\hline St. stylaris & $38 \quad(397,10.4)$ & $56.2(223,397)$ \\
\hline T. bissectus & $86 \quad(684,7.95)$ & $56.5(375,664)$ \\
\hline
\end{tabular}


TABLE 10

CONCENTRATION OF FIBER STRANDS IN INTERRIDGE AREAS*

\begin{tabular}{lccc}
\hline Genus & $\begin{array}{c}\text { Percentage of species with } \\
\text { low concentration }(0-19.9 / \mathrm{mm} \\
\text { width) }\end{array}$ & $\begin{array}{c}\text { Percentage of species with } \\
\text { moderate concentration } \\
20-49.9 / \text { mm width) }\end{array}$ & $\begin{array}{c}\text { Percentage of species with } \\
\text { high concentration }(50-109.9 / \text { mm } \\
\text { width) }\end{array}$ \\
\hline $\begin{array}{l}\text { Asplundia }(23,94) \\
\text { Carludovica }(1,3)\end{array}$ & $13.0 \%$ & $65.2 \%$ & $21.7 \%$ \\
Cyclanthus $(1,1)$ & $100 \%$ & & $100 \%$ \\
$\bar{N} \quad$ Dicranopygium $(13,48)$ & $69.2 \%$ & $30.8 \%$ & $100 \%$ \\
Evodianthus $(1,1)$ & $33.3 \%$ & $33.3 \%$ & $33.3 \%$ \\
Ludovia $(3,3)$ & & $100 \%$ & $42.9 \%$ \\
Schultesiophytum (1, 1) & & $57.1 \%$ & $100 \%$ \\
Sphaeradenia $(7,42)$ & & $100 \%$ & $100 \%$ \\
Stelestylis $(2,4)$ & & & \\
Thoracocarpus $(1,1)$ & & & \\
\hline
\end{tabular}

*In parentheses after the name of each genus are indicated the number of species considered and the total number of species per genus, respectively (not including undescribed species presently studied). 
TABLE 11

DATA PERTAINING TO FIBER STRANDS OF THE MESOPHYLL

\begin{tabular}{lcccc}
\hline & $\begin{array}{c}\text { Percentage of fiber } \\
\text { strands on both } \\
\text { sides of lamina } \\
\text { which are in sub- } \\
\text { epidermal layers* }\end{array}$ & $\begin{array}{c}\text { Percentage of adaxially } \\
\text { situated fiber strands in } \\
\text { adaxial subepidermal } \\
\text { layer* }\end{array}$ & $\begin{array}{c}\text { Percentage of abaxially } \\
\text { situated fiber strands in } \\
\text { abaxial subepidermal } \\
\text { layer* }\end{array}$ & $\begin{array}{c}\text { Mean numbers of hypo- } \\
\text { dermal parenchyma cells } \\
\text { intervening between adjacent } \\
\text { pairs of fiber strands in } \\
\text { adaxial subepidermal layer** }\end{array}$ \\
\hline A. pycnantha & $43.4(334)$ & $46.8(190)$ & $38.9(144)$ & $2.38(50)$ \\
Ca. palmata & $43.8(482)$ & $53.8(296)$ & $28.0(186)$ & $1.70(50)$ \\
Cy. bipartitus & $53.1(49)$ & $54.2(48)$ & $0(1)$ & 10.4 \\
D. crinitum & $31.9(329)$ & $34.2(184)$ & $29.0(145)$ & $4.72(50)$ \\
E. funifer & $79.9(289)$ & $82.7(173)$ & $75.9(116)$ & $1.46(50)$ \\
L. lancifolia & $48.5(482)$ & $48.4(275)$ & $48.8(207)$ & $1.40(50)$ \\
Sch. chorianthum & $75.4(187)$ & $68.3(123)$ & $89.1(64)$ & $3.68(40)$ \\
Sph. killipii & $83.1(455)$ & $79.9(229)$ & $86.3(226)$ & $1.06(50)$ \\
St. stylaris & $86.4(397)$ & $88.3(223)$ & $83.9(174)$ & $1.06(50)$ \\
T. bissectus & $29.1(663)$ & $38.0(371)$ & $17.8(292)$ & $2.14(50)$ \\
\hline
\end{tabular}

*In parentheses are indicated number of fiber strands observed.

** In parentheses are indicated number of fiber strand pairs observed. 
(Wilder, in press $c$ )

Within interridge areas and between boundary layers cyclanthaceous laminae exhibit raphide sacs and, sometimes, also styloid sacs and/or sacs intermediate between raphide and styloid sacs (Tables 12, 13). These crystal sacs normally lack chloroplasts, but at least sometimes contain leucoplasts and, apparently, normal nuclei. In raphide sacs the raphides usually comprise an orderly array, and are sometimes compound. Styloid sacs occur in Evodianthus funifer and all species studied of Sphaeradenia and Stelestylis, but are less common among remaining species of Carludovicoideae. In almost all species raphide and styloid sacs tend to be oriented along paradermal planes; however, in E. funifer the styloid sacs normally lie in all directions within the mesophyll. Scattered regions of periderm occur in various species, apparently, because of wounding. I have developed a concept of boundary layers. A boundary layer separates ordinary mesophyll tissue from another part of the plant. Cyclanthaceous laminae exhibit four types of boundary layers, viz., hypodermis, bundle sheath, epithelium of mucilage cavities, and laticifer sheath. All boundary layers exhibit significant features in common, in addition to position, including aspects of intercellular spaces between their constituent cells, and chloroplast position. In the adaxial hypodermis the shapes of hypodermal parenchyma cells in surface view are very predictable. 
TABLE 12

KINDS OF CRYSTAL SACS PRESENT WITHIN INTERRIDGE AREAS

OF CYCLANTHACEOUS LAMINAE,

\begin{tabular}{|c|c|c|c|c|}
\hline Species & 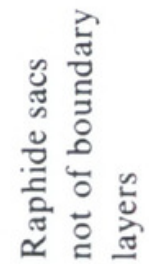 & 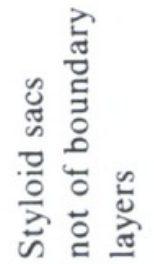 & 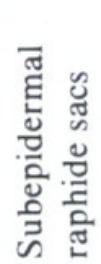 & 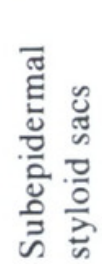 \\
\hline
\end{tabular}

A. aff. A. antioquiae (coll. A) +

A. aff A. antioquiae (coll. B) +

A. cabrerae +

A. cayapensis $\quad+(\mathrm{I}) \quad+(\mathrm{I})$

A. sp. nov. aff. A. cupulifera (coll. A)

A. sp. nov. aff. A. cupulifera (coll. B)

A. gamotepala

A. hookeri

A. longitepala

A. sp. nov. aff. A. longitepala

A. moritziana

A. aff. A. moritziana

A. sp. nov. aff. A. multistaminata

A. peruviana

A. pycnantha

A. quinindensis

$A$. sp. nov. aff. $A$. rhodea

A. rigida

A. tetragona

A. urophylla

A. aff. A. vaupesiana (coll. A)

A. aff. A. vaupesiana (coll. B)

A. (Asplundia) sp. nov.

Ca. palmata

Cy. bipartitus

$+(\mathrm{S}) \quad+$

+
+

+

$+\quad+$

$+$

$+(\mathrm{I}) \quad+(\mathrm{I}) \quad+$

$+$

$+$

$+$

$+$

$+$

$+$

$+(\mathrm{S})$

$+$

$+\quad+$

$+\quad+$

$++$

$+$

$+$

$+$

$+$

$\begin{array}{llll}+(\mathrm{I}) & +(\mathrm{I}) & + & + \\ + & +(\mathrm{Bu}) & + & \end{array}$


TABLE 12 (Continued)

KINDS OF CRYSTAL SACS PRESENT WITHIN INTERRIDGE AREAS

OF CYCLANTHACEOUS LAMINAE.

\begin{tabular}{|c|c|c|c|c|}
\hline Species & 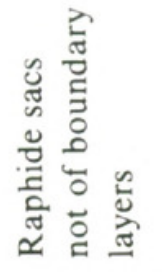 & 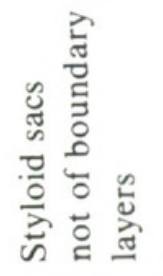 & 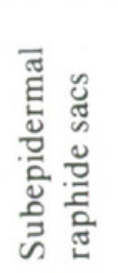 & 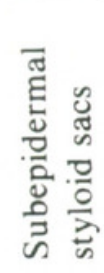 \\
\hline D. crinitum & + & & + & \\
\hline D. dolichostemon & $+(\mathrm{I})$ & $+(\mathrm{I})$ & + & \\
\hline D. globosum & + & & + & \\
\hline D. grandifolium & + & & & \\
\hline D. harlingii & + & & + & \\
\hline D. macrophyllum & + & & + & \\
\hline D. mirabile & + & & & \\
\hline D. sp. nov. aff. D. nanum & $+(\mathrm{S})$ & & + & \\
\hline D. rheithrophilum & + & & + & \\
\hline D. schultesii & + & & & \\
\hline D. wallisii & + & & & \\
\hline$D$. sp. nov. (coll. $A$ ) & + & & & \\
\hline D. sp. nov. (coll. B) & + & & + & \\
\hline E. funifer & + & + & & + \\
\hline L. bierhorstii & + & & + & \\
\hline L. integrifolia & + & & + & \\
\hline L. lancifolia & + & & + & \\
\hline Sch. chorianthum & + & & & \\
\hline Sph. acutitepala & + & + & & + \\
\hline Sph. crocea & + & + & & + \\
\hline Sph. killipii & + & + & + & \\
\hline Sph. snidernii & + & + & & \\
\hline Sph. woodsonii & + & + & & + \\
\hline Sph. sp. nov. aff. Sph. woodsonii & + & + & & \\
\hline Sph. sp. nov. & + & + & + & \\
\hline St. anomala & + & + & & + \\
\hline
\end{tabular}


TABLE 12 (Continued)

KINDS OF CRYSTAL SACS PRESENT WITHIN INTERRIDGE AREAS OF CYCLANTHACEOUS LAMINAE.

\begin{tabular}{|c|c|c|c|c|}
\hline Species & 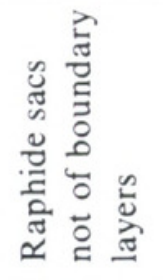 & 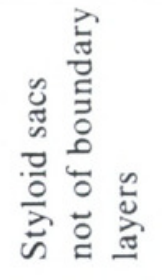 & 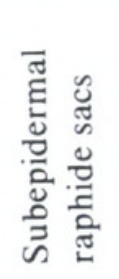 & 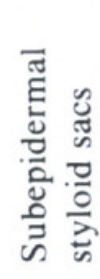 \\
\hline St. stylaris & + & + & & + \\
\hline T. bissectus & + & & & \\
\hline
\end{tabular}

$+=$ present and well-defined.

$+(\mathrm{I})=$ typical raphide sacs, styloid sacs, and all intermediates between these two cell types are present.

$+(\mathrm{S})=$ some sacs have small numbers of crystals which mostly tend to be intermediate between raphides and styloids, but well-defined styloids are generally absent.

$(\mathrm{Bu})=$ styloid sacs are mainly limited to bundle sheaths, where they occur together with raphide sacs and intermediate types of sacs. 
TABLE 13

LENGTHS OF RAPHIDE BUNDLES AND STYLOIDS IN CLEARED LAMINAE ( $\mu$ M, MEASURED USING CROSSED POLARS)

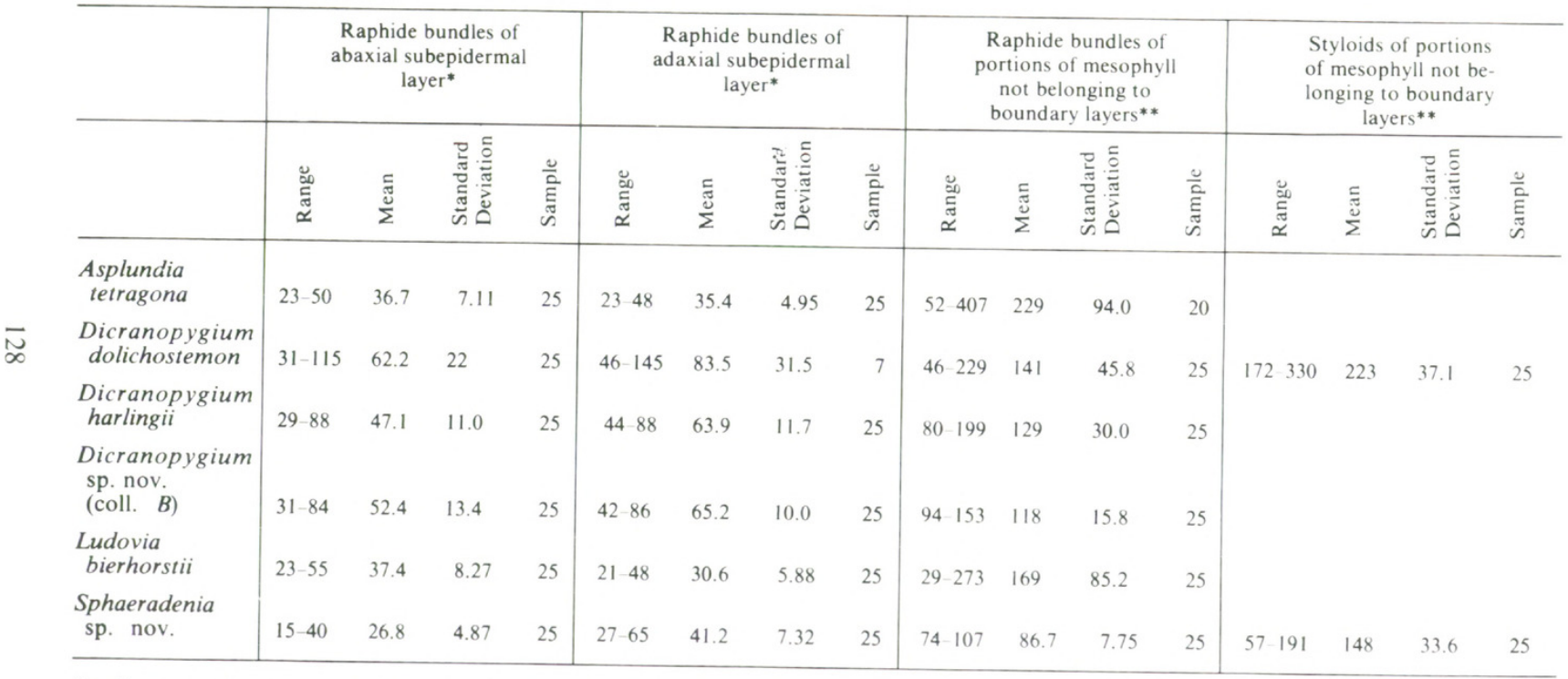
*In $D$. sp. nov. bundles of the adaxial and abaxial hypodermides are commonly oriented anticlinally or nearly so; only bundles with essentially paradermal
orientations were measured.

**In $D$. dolichostemon with all intermediates between raphides and styloids, crystals were measured only if they were clearly raphides (very narrow crystals, generally hypodermis were oriented anticlinally or nearly so, and were not measured. 
Interridge areas of cyclanthaceous laminae exhibit longitudinal veins and commissural veins which vary from transverse to oblique. In at least some species of the Asplundia group, but not all of the Sphaeradenia group, these longitudinal veins tend to be oriented nearest the abaxial surface of the lamina (Table 14). In many species longitudinal veins of interridge areas are of discrete orders, and up to a given order the number of veins of an order is twice, or nearly twice that of the next lower order (Table 15). Whereas, longitudinal veins of interridge areas are normally upright, commissures vary from upright to inverted. In ten species cleared portions of lamina each measuring $120 \mathrm{~mm}^{2}$ exhibited from six to forty-seven commissures (Table 16). Expansion tissue and presumed expansion tissue develop in all species of Carludovicoideae and in Cyclanthus bipartitus, respectively. Adaxial and abaxial ridges are mostly associated with one or more longitudinal veins. The main vein of a ridge is normally upright, whereas, additional vein(s) may be upright or inverted to various degrees. In interridge areas and ridges longitudinal veins are normally collateral, but bicollateral, amphivasal, and amphicribal veins may also be present. 


\section{TABLE 14}

POSITIONS OF LONGITUDINAL VEINS IN INTERRIDGE AREAS (CARLUDOVICOIDEAE) OR NONCOSTAL PORTIONS OF LAMINA (CYCLANTHOIDEAE) RELATIVE TO THE ADAXIAL AND ABAXIAL SURFACES OF THE LAMINA *

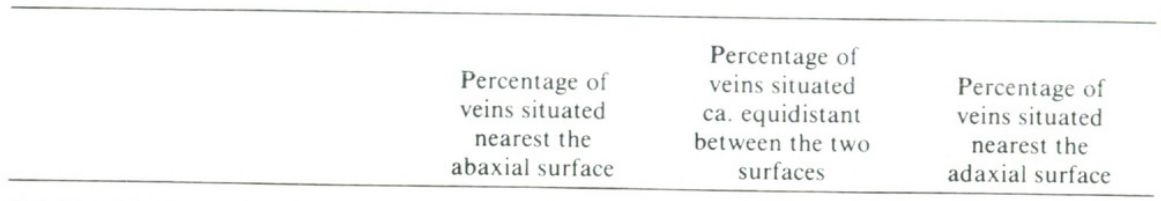

\section{CYCLANTHOIDEAE}

Cyclanthus bipartitus

(20)

\section{CARLUDOVICOIDEAE}

\section{ASPLUNDIA GROUP}

A. pycnantha $(20)$

100

Ca. palmata $(20)$

100

D. crinitum (20)

100

E. funifer (20)

Sch. chorianthum (18)

T. bissectus (20)

SPHAERADENIA

GROUP

L. lancifolia (16)

Sph. killipii (18) 
TABLE 15

MEAN PERCENTAGES OF THE EXPECTED NUMBERS OF LONGITUDINAL VEINS OF EACH ORDER PRESENT IN THE INTERRIDGE AREAS OF TWENTY-TWO SPECIES OF ASPLUNDIA, TEN OF DICRANOPYGIUM, AND FOUR OF SPHAERADENIA, AND STANDARD DEVIATIONS.***

\begin{tabular}{|c|c|c|c|c|c|c|c|}
\hline & $\begin{array}{l}\text { First } \\
\text { Order }\end{array}$ & $\begin{array}{l}\text { Second } \\
\text { Order }\end{array}$ & $\begin{array}{l}\text { Third } \\
\text { Order }\end{array}$ & $\begin{array}{l}\text { Fourth } \\
\text { Order }\end{array}$ & $\begin{array}{l}\text { Fifth } \\
\text { Order }\end{array}$ & $\begin{array}{l}\text { Sixth } \\
\text { Order }\end{array}$ & $\begin{array}{c}\text { Seventh and } \\
\text { Higher Orders }\end{array}$ \\
\hline Asplundia (29) & 100,0 & 100,0 & $98.9,5.33$ & $71.9,33.4$ & $15.3,21.6$ & $0.43,1.46$ & 0,0 \\
\hline $\begin{array}{l}\text { Dicranopygium } \\
\quad(15.5)\end{array}$ & 100,0 & $98.3,5.28$ & $90,19.2$ & $48.3,37.9$ & $5.63,9.41$ & 0,0 & 0,0 \\
\hline Sphaeradenia (4) & 100,0 & 100,0 & 100,0 & $68.8,33.1$ & $35.9,35.5$ & $1.56,1.80$ & 0,0 \\
\hline
\end{tabular}


TABLE 16

NUMBERS OF COMMISSURES WITHIN $120 \mathrm{~mm}^{2}$ OF LAMINA, AWAY FROM COSTAE*

\begin{tabular}{lc} 
SPECIES & NUMBER OF COMMISSURES \\
\hline A. pycnantha & 10 \\
Ca. palmata & 31 \\
Cy. bipartitus & 31 \\
D. crinitum & 47 \\
E. funifer & 12 \\
L. lancifolia & 10 \\
Sch. chorianthum & 45 \\
Sph. killipii & 10 \\
St. stylaris & $14-15$ \\
T. bissectus & 6
\end{tabular}

${ }^{*}$ Branches of commissures were counted as separate commissures. For example, in a case where a commissure became divided into two parts at one end, the undivided portion and its two products were interpreted to represent a total of two commissures.

\section{ACKNOWLEGMENTS}

Initial stages of this study were done under a postdoctoral fellowship provided by the Cabot Foundation, Harvard University, and a concomitant appointment at the Fairchild Tropical Garden, Miami, Florida. Funds were also obtained from National Science Foundation grants BSR-82-10618, 78-04731, and GB31844X. Additional support for travel came from the Atkins Funds and Cabot Foundation of Harvard University and the Research Board of the University of Illinois at Chicago Circle.

I am deeply grateful to Dr. P. B. TOMLINSON of Harvard University for his invaluable help, rendered on numerous occasions. I am indebted, too, to Dr. GUNNAR HARLING of the University of Göteborg for his encouragement and help in identifying virtually all of the species presently studied. I also express deep appreciation to the following individuals and institutions for their assistance during this investigation: Dr. JOHN POPENOE and the Fairchild Tropical Garden; Dr. ROBERT DRESSLER and the Smithsonian Tropical Research Institute; Dr. R. A. 
OWEN, Mr. RAMNARINE PERSAUD and the Forest Department of the Republic of Guyana; Dr. OMAWALE and the University of Guyana; Dr. JAIRO CORREA, Dr. JAIME RIVERA CASTRO, and the Universidad Nacional de Colombia, Medellín; Dr. ALVARO FERNANDEZ-PEREZ, Dr. ENRIQUE FORERO, and the Universidad Nacional de Colombia, Bogotá; Dr. ENRIQUE HERNANDEZ and the Universidad de Nariño, Pasto, Colombia; Dr. CIRO A. VALOYES, sectional head of Inderena, Quibdo, Colombia; Dr. JULIAN STEYERMARCK, Dr. GILBERTO MORILLO, Dr. ANGIL GONZALES, and the Instituto Botánico, Caracas, Venezuela; Dr. BASSETT MAGUIRE and the New York Botanical Garden; Dr. RICHARD SCHULTES and the Gray Herbarium, Harvard University, Mr. TOMAS QUIONES, mayor of Puerto Nariño, Colombia; Dr. WILLIAM BURGER and the Field Museum of Natural History.

In addition, the following individuals provided indispensable assistance: Mrs. REBECCA WILDER; Mr. JAIME MORENO; Mr. DENNIS MAGEE; Mr. EDUARDO CALDERON; MR. DANIEL HARRIS; Mrs. HELEN WOODEN; Mr. JEFFREY VINCENT; Ms. DOROTHY SMITH; and IGNACIO, ALVARO, CAROLYN, and DIEGO VILLA. Mr. WILLIAM ORMEROD duplicated my color transparencies with black-andwhite film, and prepared prints; he also made the original photographs of specimens in figs. 1-4 in Wilder (in press $d$ ).

\section{LITERATURE CITED}

Tomlinson, P. B., and G. J. Wilder. 1984. Systematic anatomy of Cyclanthaceae (Monocotyledoneae) - an overview. Bot. Gaz.: in press.

Wilder, G. J. Anatomy of noncostal portions of lamina in the Cyclanthaceae (Monocotyledoneae). I. Epidermis. Bot. Gaz.: in press $a$.

Anatomy of noncostal portions of lamina in the Cyclanthaceae (Monocotyledoneae). II. Regions of mesophyll, monomorphic and dimorphic ordinary parenchyma cells, mesophyll fibers, and parenchyma-like dead cells. Bot. Gaz.: in press $b$.

Anatomy of noncostal portions of lamina in the Cyclanthaceae (Monocotyledoneae). III. Crystal sacs, periderm, and boundary layers of the mesophyll. Bot. Gaz.: in press $c$.

Anatomy of noncostal portions of lamina in the Cyclanthaceae (Monocotyledoneae). $I V$. Veins of interridge areas, expansion tissue, and adaxial and abaxial ridges. Bot. Gaz.: in press $d$. 


\section{$2 \mathrm{BHL}$ Biodiversity Heritage Library}

Wilder, George J. 1985. "Anatomy of Noncostal Portions of Lamina in the Cyclanthaceae (Monocotyledoneae). V. Tables of Data." Botanical Museum leaflets, Harvard University 30(2), 103-133. https://doi.org/10.5962/p.168675.

View This Item Online: https://www.biodiversitylibrary.org/item/31877

DOI: https://doi.org/10.5962/p.168675

Permalink: https://www.biodiversitylibrary.org/partpdf/168675

\section{Holding Institution}

Missouri Botanical Garden, Peter H. Raven Library

\section{Sponsored by}

Missouri Botanical Garden

\section{Copyright \& Reuse}

Copyright Status: Public domain. The BHL considers that this work is no longer under copyright protection.

This document was created from content at the Biodiversity Heritage Library, the world's largest open access digital library for biodiversity literature and archives. Visit BHL at https://www.biodiversitylibrary.org. 